\title{
Health seeking behaviour and access to care for mobile and migrant populations in Cambodia
}

Soy Ty Kheang ${ }^{1,2 *}$, Emma Louise Collins ${ }^{1}$, Ashley Preston ${ }^{1}$, Yom An ${ }^{2}$, Por Ir ${ }^{3}$, Hok Phalla ${ }^{3}$, Dysoley Lek ${ }^{3,4}$, Rekol Huy ${ }^{4}$, Sovannaroath Siv ${ }^{4}$ Hala J AlMossawi ${ }^{5}$ and Neeraj Kak

${ }^{1}$ Health and Social Development (HSD), Phnom Penh, Cambodia

${ }^{2}$ University Research Co., LLC (URC), Phnom Penh, Cambodia

${ }^{3}$ School of Public Health, National Institute of Public Health (NIPH), Cambodia

${ }^{4}$ National Centre for Parasitology, Entomology and Malaria Control, Cambodia

${ }^{5}$ Centre for Innovations and Technology, University Research Co., LLC (URC), USA

\begin{abstract}
Background: Malaria elimination campaigns are making huge strides in Cambodia with a target of malaria elimination by 2025 . However, pockets of transmission remain. Predominately in border forested areas in Western Cambodia, the populations residing here are often part of mobile and migrant populations (MMPs). Moreover, the rise of drug resistance makes the goal of malaria elimination ever more urgent.

Materials and methods: The Cambodia Mobile and Migrant Population Survey was a cross-sectional survey aimed to gain further insight into MMPs to inform malaria control and elimination interventions. A purposive sampling technique was used to identify study participants, given the difficulty of identifying and accessing MMPs. The survey was conducted across ten malaria endemic health districts in 2017. Separation of MMPs into four main occupational subgroups; construction workers, seasonal workers, forest goers and security personnel, was used for analysis.

Results: The results from this study confirm MMPs are a key group at higher risk of malaria due to their associated risky behaviours. MMPs have a significantly different risk of suffering from fever between subgroups with forest goers and seasonal workers far more likely to report suffering from fever within the last three months. However, the willingness to seek healthcare because of fever was remarkably good, and similar among subgroups. A concerning finding was the low numbers of suspected malaria cases being diagnosed with parasitological blood testing and just under half of MMPs were not aware that malaria should be confirmed by parasitological blood test.
\end{abstract}

Conclusion: MMPs are at an elevated risk of malaria compared to the general population, and this risk varies within MMPs depending on the subgroup. Although health seeking behaviour was reportedly good for all MMP subgroups to public health facilities and volunteers, barriers in access to care were significantly different. This highlights that MMPs can no longer be treated as a homogenous group when targeted by malaria interventions.

\section{Background}

The past decade has seen impressive improvements in malaria control, presenting the possibility for renewed attempts for elimination [1]. Cambodia made huge strides in reducing malaria, and over the last decade, cases caused by Plasmodium falciparum have reduced by $81 \%$ [2]. As a result, Cambodia aims to completely eliminate malaria by 2025 [3]. However, the emergence and spread of $P$. falciparum artemisinin drug resistance poses major challenges to achieving these goals as treatment failure becomes increasingly common $[4,5]$. Resistance was first reported on the Thai-Cambodia border in 2008 and has since spread to several more countries in the Greater Mekong Sub-region (GMS) causing increasing concern for global malaria mortality rates $[6,7]$. Features of the current social landscape in Cambodia, including having a highly mobile population with reduced access to public healthcare, and the high utilisation of unregulated private healthcare, may further exacerbate the existing problems of resistance [8].

In the setting of elimination, the remaining cases often cluster in particular groups, generally those who are most vulnerable and marginalised, who engage in high risk behaviours $[9,10]$. It is these foci which therefore need to be understood best to achieve elimination. In Cambodia, malaria transmission tends to be concentrated in Mobile and Migrant Populations (MMPs). MMPs have been found to be three times more likely to have clinical malaria episodes compared to the general population, and in the Thailand-Cambodia border regions, areas with greater numbers of migrants also had correspondingly higher rates of malaria $[11,12]$. Due to the high mobility of MMPs, they often do not have access to routine surveillance or health promotion initiatives and they are more likely to seek more accessible unlicensed private healthcare, as was found to be the case in MMPs in Myanmar [13-16]. Thus the characteristics of this population are suggested to be one of the main drivers for malaria transmission and spread of drug resistance $[5,16,17]$. It is feared that this could lead to the reintroduction of malaria into previously malaria-free zones, especially as cases are often reported when migrants have returned to their home residence for treatment $[17,18]$. Migration poses significant challenges to the elimination of malaria and this will not be effectively dealt with until MMPs are better understood.

*Correspondence to: Soy Ty Kheang, University Research Co., LLC, Phnom Penh, Cambodia, Tel: +855 17988 388; E-mail: ksoyty@urc-chs.com

Received: June 10, 2019; Accepted: June 24, 2019; Published: June 27, 2019 
In Cambodia, the generally accepted definition of MMPs are that mobile individuals have been residing in the area for less than six months and migrant individuals have been there for more than six months, but less than a year $[17,19]$. MMPs are at greater risk of malaria than the general population for several key reasons; one of the main reasons is that their work is related to the forest, putting them at high risk of coming into contact with malaria vectors, and this forestrelated transmission is the most common form of transmission in Cambodia $[15,17,20]$. Multiple studies have shown that significantly higher parasite rates have been found in migrants and villagers living near forest areas compared to the general population $[21,22]$. Other key reasons include the health seeking behaviour of MMPs and the barriers they face when accessing care. A study focusing on migrants working on the Thailand-Cambodia border found that between 15-27\% (depending on the migrant subgroup) would not seek treatment for suspected malaria [23]. If MMPs do seek healthcare, they are likely to seek private healthcare providers, potentially increasing their exposure to poor quality drugs and treatment regimens [14,20]. The most important determinants for MMPs to seek care were the proximity of services and the cost, including cost of treatment and also transport to get there $[8,23]$. Furthermore, they often have poor access to healthcare as they work in remote areas, making them more likely to have late diagnosis and they are often difficult to follow up to ensure treatment compliance and clearance of parasites [5,18]. Key factors influencing MMP access to healthcare in the GMS included geographical constraints, barriers to do with culture or language, financial constraints and potential eligibility or legal restrictions for migrant health [5,24].
Although the general characteristics of MMPs are understood, interventions often target them as a homogenous group; however, without understanding the unique behaviours of each subgroup within MMPs, they will never be effectively targeted. Health seeking behaviour and barriers to healthcare access were identified as key research priorities to develop malaria control interventions $[4,18]$. Therefore, the aim of this study is to create a detailed picture of the health seeking behaviour of the key MMP subgroups at risk of malaria in Cambodia.

\section{Materials and methods}

\section{Study design}

This study is a subset of the Cambodia Mobile and Migrant Population Survey 2017, therefore following the same study design, but purely focusing on health seeking behaviour. The survey was a cross-sectional non-probability sampling survey of MMPs using questionnaires. This was undertaken between March and April 2017.

\section{Study site and population}

Ten malaria endemic operational districts (ODs) within the provinces of Pursat (Kravanh, Krakor), Battambang (Maung Russei, Sampov Loun, Battambang), Oddar Meanchey (Somrong, Anlong Veng), Stung Treng (Stung Treng) and Rattanak Kiri (Banlong, Borkeo) were selected for this study based on their geographical and epidemiological situation (Figure 1). The survey sites within these districts were selected based on a field assessment conducted prior to data collection. The locations and population size of MMPs were

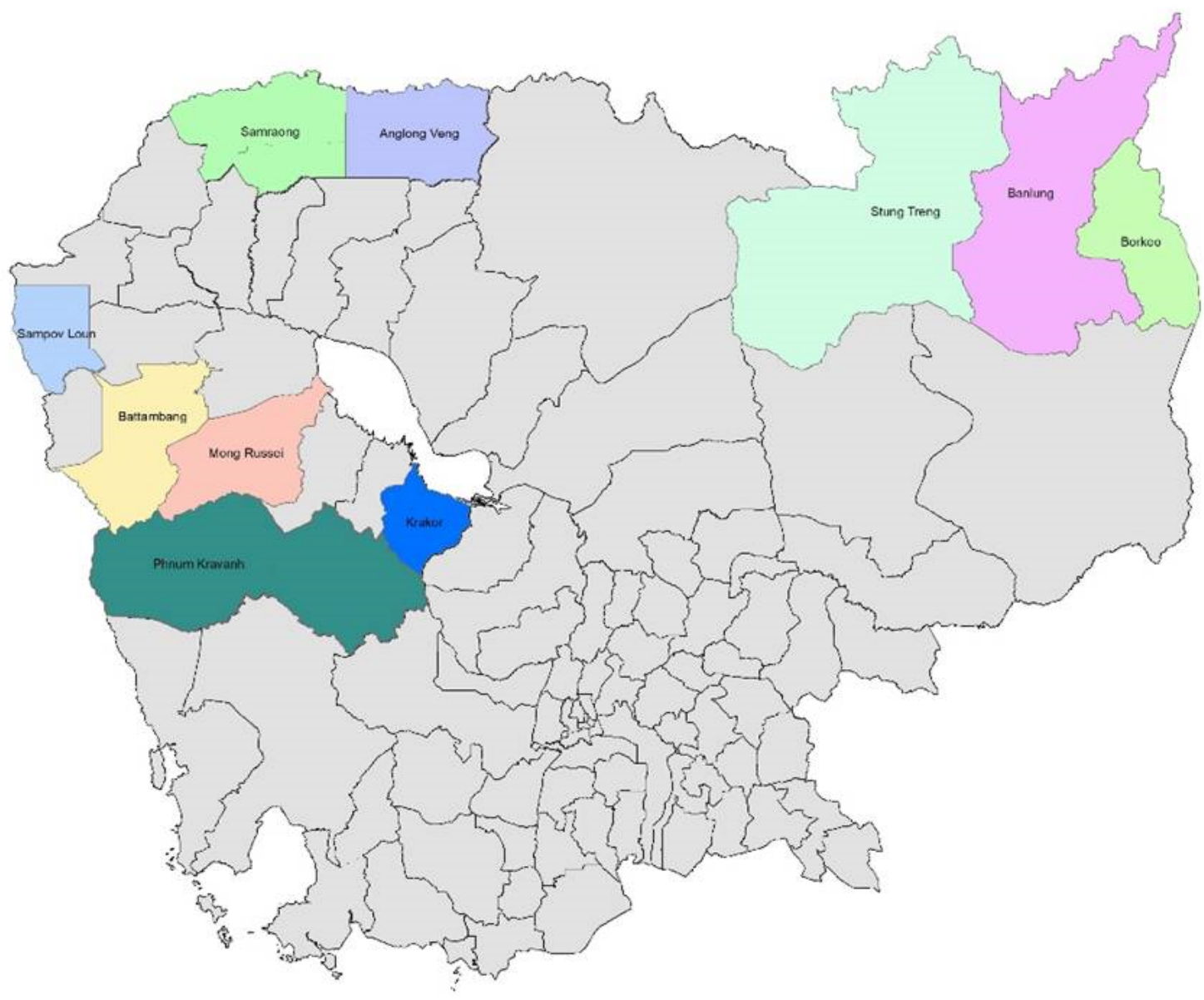

Figure 1. Operational districts covered by the survey. 
estimated with the aid of key informants including Village Malaria Workers (VMWs), the OD director, the OD malaria supervisor, some health centre chiefs, and the village chiefs. Information collected was used to guide selection of survey sites and data collection procedures.

MMP definitions for this study were two-fold: migrant populations encompass people who have moved their place of residence within the last 6-12 months; and mobile populations have moved their place of residence in the last 6 months. MMPs were further sub-categorised by their profession $[17,19]$ :

- Seasonal workers involved in agriculture activities during the planting season and the harvesting season are usually localized in foothills/plains/valleys;

- Construction/mine workers related to construction, mining, dam and demining activities are usually found in foothills/plains/valleys;

- Forest goers which include not only individuals engaged in mobile or migrant movements but also local residents who engage in circular migration into forested areas;

- Security personnel including military and police often related to patrolling activities in forested border areas.

\section{Sampling}

A purposive sampling technique was used to identify study participants, given the difficulty of identifying and accessing MMPs. An even distribution was selected over the four subgroups of MMPs over the ten operational districts selected. The sample size was calculated, with a desired $95 \%$ confidence interval, to be 1,200 individuals for each MMP subgroup and an overall sample size of 4,800.

\section{Ethical considerations}

The survey was approved by the National Ethics Committee for Health Research in Cambodia (reference number: 065 NECHR). Prior to participating in the study, written consent was obtained from each participant, ensuring they fully understood the nature of the survey and that is was confidential.

\section{Data collection}

A field guide was developed to provide instructions on the best practice for field data collection, including how to select appropriate survey sites and eligible MMP candidates. The guide also included the assigned tasks for all members of the data collection team: data collectors, field supervisors, and field team leaders. They were all given a thorough one-day training on their role and to ensure high quality data collection, trained personnel could refer to the field guide, if necessary. There were five data collection teams, each made up of five trained personnel, including four data collectors and one field supervisor. All field work was managed by two field team leaders, with supervision from a senior researcher from the National Institute of Public Health.

Due to the hard to reach nature of MMPs, they were selected through a range of access points. The majority were accessed through their place of work, namely plantations and agricultural farms for seasonal workers, mine or construction sites for construction workers, policy or military camps for security personnel, and at-risk villages close to the forest for forest goers.

A structured questionnaire was designed for data collection and included seven sections. The complete questionnaire is available upon request; this paper focuses on Section 1 which collected demographic information, Section 5 which focused on fever, health seeking behaviour and parasite-based diagnosis in the last 3 months, and Section 7 which covered knowledge of malaria risk and prevention. The questionnaire was then translated into the local language Khmer and was piloted to ensure the meaning of questions was clear and had not changed due to translation. The pilot was also used to train data collectors and allow them to familiarise themselves with the process.

\section{Data analysis}

Completed questionnaires were collected by field supervisors and checked for completeness and accuracy. The data was double entered and collated into a standardised database form in Excel, the dataset was then cleaned by a senior researcher. Further analysis for this paper was conducted using the statistical software SPSS (IBM Corp. Released 2013. IBM SPSS Statistics for Windows, Version 22.0. Armonk, NY: IBM Corp). Analysis on key themes was conducted using Chi-square tests to compare proportions between MMP subgroups and significance was determined at the $5 \%$ level $(p<0.05)$. The $95 \%$ confidence intervals (CI) are also stated where appropriate.

\section{Results}

\section{Socio-demographics of sample}

In total 4,844 MMPs completed the questionnaire, made up of 1,209 seasonal workers, 1,215 construction/mine workers, 1,210 forest goers, and 1,210 security personnel. The majority of MMPs were male, although the distribution was more even in seasonal workers (Table 1). The mean age of MMPs was 35 years (ranging from 18 to 83 years) and this was similar across all subgroups.

The majority of MMPs either lived with family or co-workers and this varied by subgroup. Those who were more likely to live with their family included seasonal workers $(78.3 \%$ ) and forest goers (having been selected from their village residence, $95.1 \%)$. In contrast, construction/ mine workers and security personnel were more likely to live with coworkers ( $53.5 \%$ and $66.9 \%$, respectively). Out of all respondents, 2,858 (59.0\%) reported to have lived with at least one other family member, the majority of these either lived with one (21.6\%), two (27.1\%) or three (21.5\%) other family members. Of the 2,858 respondents living with their families, $45.0 \%$ had at least one boy or one girl under five years old living in their household.

\section{Health seeking behaviour between MMP subgroups}

In the survey population $37 \%$ reported that they had suffered from fever in the last three months, and this differed between subgroups, as seen in figure 2, with forest goers and seasonal workers more likely to suffer from fever compared to construction/mine workers and security personnel. This difference was statistically significant $\left(\mathrm{X}^{2}=11.98, \mathrm{df}=3\right.$, $p=0.007)$.

Of the 1,770 reporting fever in the last three months, health seeking behaviour was good with $88.9 \%$ (95\% CI 87.4 -90.3) seeking medical advice or treatment. Between each MMP subgroup, the proportion seeking care varied slightly; forest goers had the highest proportion at 92\% (95\% CI 90.1 - 94.8), then $89.8 \%$ (95\% CI 86.8 - 92.7) in security personnel, $88.5 \%$ (95\% CI 85.4 - 91.6) in construction/mine workers, and the lowest was reported for seasonal workers at $84.6 \%$ (95\% CI 81.3 - 87.9). However, the difference between subgroups was not statistically significant $\left(\mathrm{X}^{2}=1.698, \mathrm{df}=3, p=0.64\right)$. 
Table 1. Distribution of Gender within each MMP Subgroup.

\begin{tabular}{|c|c|c|c|c|c|}
\hline Gender & $\begin{array}{c}\text { Seasonal workers }(\%) \\
(\mathrm{N}=1,209)\end{array}$ & $\begin{array}{c}\text { Construction / mine } \\
\text { workers }(\%)(N=1,215)\end{array}$ & Forest goers $(\%)(\mathrm{N}=1,210)$ & $\begin{array}{l}\text { Security personnel (\%) } \\
(\mathrm{N}=\mathbf{1 , 2 1 0})\end{array}$ & $\begin{array}{l}\text { All subgroups } \\
(\%)(\mathrm{N}=4,844)\end{array}$ \\
\hline Male & 48.7 & 80.7 & 69.7 & 98.7 & 74.4 \\
\hline Female & 51.3 & 19.3 & 30.3 & 1.3 & 25.6 \\
\hline
\end{tabular}

\section{Proportion of Fever Suffered by Different MMP Subgroups}

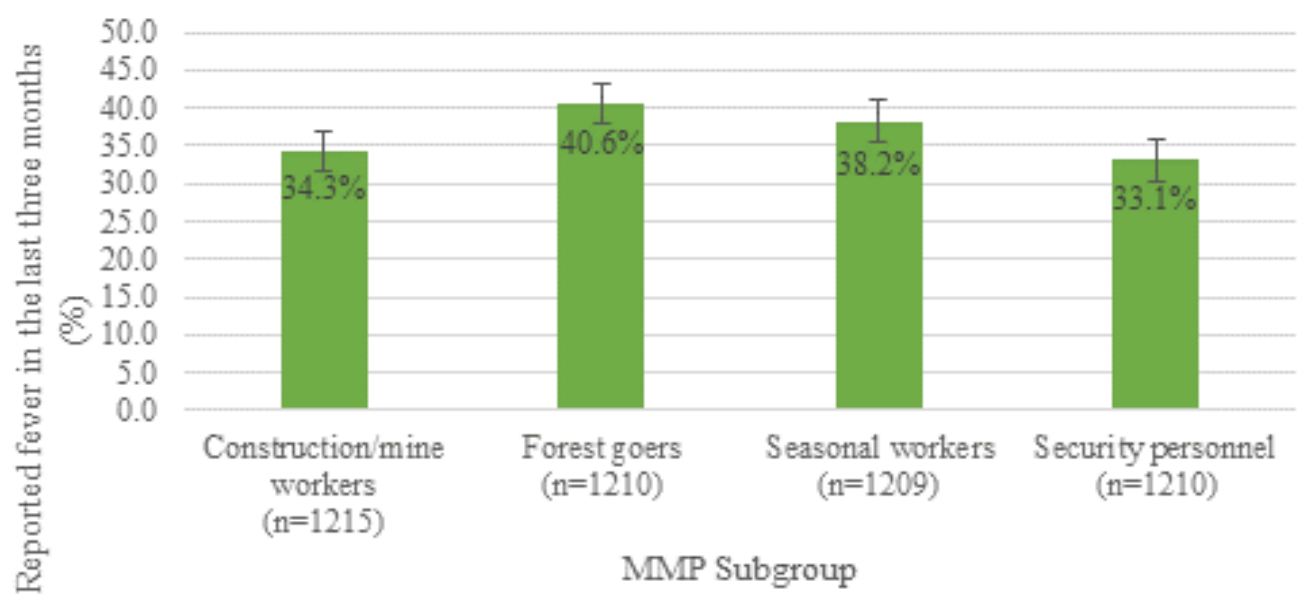

Figure 2. Reported fever in the last three months (\%) by MMP subgroup. The $95 \%$ confidence intervals (CIs) are represented; they indicate that the proportion of fever suffered by forest goers was significantly greater compared to construction/mine workers and security personnel.

\section{Seeking healthcare in MMPs was significantly associated with their family members also seeking healthcare}

Of the 2,858 MMPs who lived with family members, $56.8 \%$ (95\% CI 54.9 - 58.6) had at least one family member who had also suffered from fever in the last three months. This varied between MMP subgroups, with forest goers' family members suffering the most fever $(62.0 \%, 95 \%$ CI 59.2 - 64.8), followed by seasonal workers (58.3\%, 95\% CI 55.1 $61.4)$, security personnel $(52.8 \%, 95 \%$ CI 47.5 - 58.2) and construction workers $(42.1 \%, 95 \%$ CI 37.4 - 46.8). This followed a similar pattern to reported fever in MMPs themselves. The majority of MMPs had only one other family member reported to have fever $(63.0 \%, \mathrm{n}=1,622)$. However, forest goers, seasonal workers and security personnel were far more likely to have had two or more family members who suffered from fever $(42.8 \% \mathrm{n}=710,33.6 \% \mathrm{n}=551,43.5 \% \mathrm{n}=177$, respectively) compared to construction workers (only $18.4 \%, \mathrm{n}=179$ ).

Again, health seeking was excellent, reportedly even better than MMPs, with 94.6\% (95\% CI 93.6-95.7) seeking advice or treatment. Health seeking behaviour between MMPs and their family members was significantly associated suggesting that if MMPs reported to seek advice or treatment, their family members would also seek healthcare services $\left(\mathrm{X}^{2}=1173536.66, \mathrm{df}=4, p<0.001\right)$.

\section{MMPs have poor access to appropriate malaria diagnosis} with parasitological blood testing

Of the 1,573 who sought medical advice or treatment, only $32.5 \%$ (95\% CI 30.2 - 34.9) received a parasitological blood test. This was even lower in their families (26.3\%; 95\% CI 24.2 - 28.4). Of those MMPs receiving testing, $40.2 \%(95 \% \mathrm{CI} 36.0$ - 44.5) reported that they received a positive malaria result. This suggests that the shortage of testing could result in as many as 427 cases of malaria not being diagnosed in the survey population (calculation: $1061 \mathrm{X}$ 0.402). Of those who were tested and found to be positive, all of them were treated (although the treatment was not specified and therefore it is not known if effective treatment was obtained).
Among MMP subgroups, there was significant variation in the number who received appropriate malaria diagnosis with parasitological blood testing $\left(\mathrm{X}^{2}=37.112, \mathrm{df}=3, p<0.001\right)$. Forest goers were the most likely to receive a blood test $(43.6 \%$; $95 \%$ CI 39.1 - 48.2), closely followed by security personnel $(37.0 \%$; 95\% CI $32.1-42.0)$, whereas this was much less likely for both seasonal workers $(23.8 \%$; $95 \%$ CI $19.6-28.0)$ and construction/mine workers (23.8\%; $95 \%$ CI 19.5 - 28.2). A similar pattern was observed for the families of MMPs.

\section{The majority of reported testing was provided by public health services}

The health services reported most frequently to provide parasitological blood testing were health centres and former district hospitals (FDHs), making up $40 \%$ of all providers of testing in this survey. This was followed by VMWs, MMWs and VHVs, and private providers ( $21 \%$ and $19 \%$ respectively) (Table 2$)$. Public health facilities accounted for $58 \%$ in total. There was a significant association between different MMP groups and the health services they frequented which provided testing $\left(\mathrm{X}^{2}=1405046.74, \mathrm{df}=32, \mathrm{p}<0.001\right)$. As highlighted in table 2, although all MMP subgroups received testing from health centres and FDHs, there was great variation between other healthcare providers which provided testing (key discrepancies are highlighted). The total number of health providers sought is not available, therefore only those providers who offered testing are presented.

\section{MMP knowledge and perceptions may influence health seeking behaviour}

MMPs had good knowledge of the common symptoms of malaria and understood the consequences of not taking treatment appropriately. Of the 1,770 participants who had reported fever in the last three months, they had good knowledge of where to seek care, with $99.5 \%$ mentioning at least one type of healthcare provider which would offer malaria services or treatment. As seen in Table 3, the most commonly mentioned places were health centres and FDHs. Private providers, 
Table 2. Most Frequently Reported Health Services Providing Testing by MMP Subgroup.

\begin{tabular}{|c|c|c|c|c|c|}
\hline $\begin{array}{c}\text { Healthcare Provider which } \\
\text { Provided Parasitological } \\
\text { Blood Testing }\end{array}$ & $\begin{array}{c}\text { Construction/ mine } \\
\text { Workers }(\mathrm{N}=\mathbf{8 8})(\mathbf{9 5 \%} \mathrm{CI})\end{array}$ & $\begin{array}{c}\text { Forest Goers }(\mathrm{N}=198) \% \\
(95 \% \mathrm{CI})\end{array}$ & $\begin{array}{c}\text { Seasonal Workers }(\mathrm{N}=93) \% \\
(\mathbf{9 5 \%} \mathrm{CI})\end{array}$ & $\begin{array}{c}\text { Security Personnel } \\
(\mathbf{N}=133) \%(95 \% \mathrm{CI})\end{array}$ & Total $(\mathrm{N}=512) \%(95 \% \mathrm{CI})$ \\
\hline Health Centre/FDH & $44.3(33.9-54.7)$ & $36.4(29.7-43.1)$ & $44.1(34.0-54.2)$ & $39.1(22.5-55.7)$ & $39.8(35.6-44.1)$ \\
\hline Health post & $4.6(0.2-8.9)$ & $0.5(0-1.5)$ & $1.1(0-3.2)$ & 29.3(13.8-44.9) & $8.8(6.3-11.2)$ \\
\hline Private health provider & $21.6(13.0-30.2)$ & 14.7(9.7-19.6) & $31.2(21.8-40.6)$ & $15.8(3.3-28.2)$ & $19.1(15.7-22.5)$ \\
\hline VMW/MMW/VHV & $10.2(3.9-16.6)$ & 41.4(34.5-48.3) & $9.7(3.7-15.7)$ & $6.0(0-14.1)$ & $21.1(17.6-24.6)$ \\
\hline
\end{tabular}

Key: Bold = proportions which show particular variation compared between MMP subgroup.

Table 3. Reported healthcare providers who could offer malaria services.

\begin{tabular}{|l|c|c|}
\hline Healthcare Providers Sought for Malaria Services & Total Number of Times Reported & Percentage of MMPs who Reported (n=1770) \\
\hline Health center/FDH & 1416 & 50.0 \\
\hline Private health provider & 594 & 33.6 \\
\hline Referral/Provincial hospital & 458 & 25.9 \\
\hline VMW/MMW/Village volunteer & 401 & 22.7 \\
\hline Army hospital/company hospital & 142 & 5.0 \\
\hline Pharmacy & 52 & 2.9 \\
\hline Private laboratory & 24 & 1.4 \\
\hline Self-treat with drugs at home & 6 & 0.3 \\
\hline Take traditional medicine & 3 & \\
\hline Shop/market & 1 & \\
\hline Not Sure & 5 & \\
\hline
\end{tabular}

referral and provincial hospitals and VMWs, MMWs and village volunteers were also commonly mentioned. $56.5 \%$ of respondents mentioned only public providers. Similarly, MMPs in general preferred sources of health information from health facility staff, although forest goers were more likely to prefer VMWs and VHVs as a source; this indicates that MMPs want to access public health providers for health information.

Of the 1,770 reporting fever, $1,324(74.8 \%$; $95 \%$ CI $72.8-77.0)$ had heard of Rapid Diagnostic Tests (RDTs) for malaria, however this was much higher in forest goers $(83.1 \%$; 95\% CI $79.7-86.5)$ and security personnel $(81.0 \%$; 95\% CI 76.9 - 85.1) compared to seasonal workers (67.2\%; 95\% CI 62.5 - 71.9) and construction/mine workers $(68.1 \%$; 95\% CI 63.3 - 72.9). However, despite many MMPs having heard of RDTs, knowledge of how to confirm malaria was less good, only $54.6 \%$ mentioned RDTs or blood tests and microscopy (or both) as a means of testing malaria, meaning that just under half of MMPs were not aware that malaria should be confirmed by parasitological blood test. Other ways commonly mentioned to confirm malaria were a doctor's examination (14.6\%) or by symptoms only (7.3\%). Forest goers and security personnel were more likely to mention RDTs as a way to confirm malaria, and this was significantly different to seasonal workers and construction/mine workers $\left(\mathrm{X}^{2}=11.48, \mathrm{df}=3, p=0.009\right)$.

Among those who had heard of RDTs, $98.9 \%$ knew where to get them, and this did not differ between MMP subgroups. Many MMPs mentioned multiple places, the most common were health centres and FDHs (58.93\%), VMWs, MMWs and VHVs (22.88\%), private health providers (21.86\%) and referral hospitals (17.29\%). Trust for the safety and reliability of RDTs was also very high, on average $97.1 \%$ for all MMPs who had heard of them, with very little variation between subgroups.

\section{Discussion}

The global health community has identified MMPs, particularly from migration hotspots along country borders, as a key group at higher risk of malaria due to their associated risky behaviours. The results from this study confirm this, as the reported rate of malaria found was double that of the general population in endemic ODs in Cambodia [9-12]. The heterogeneity of these MMPs was highlighted by the significantly different risk of suffering from fever between subgroups; forest goers and seasonal workers were far more likely to report suffering from fever within the last three months compared to security and construction/mine workers. This highlights the need for malaria interventions to be designed with these differences in mind $[14,17]$. However, some of the key high-risk behaviours usually reported in MMPs were not identified in the surveyed population; for example, the willingness to seek healthcare as a result of fever was remarkably good, and similar among subgroups. Furthermore, MMPs showed an overall preference for public healthcare providers. In previous studies in similar populations in the GMS, preference for unregulated private providers has been a cause for concern due to an increased risk of drug resistance from poor practices $[16,20]$. The private sector was still one of the most commonly cited options for accessing malaria services in this study, but this was lower than reported health seeking in the private sector for similar MMP populations in 2009 (34\% compared to $42-45 \%$, respectively) [23]. Results from this study suggest a shift towards MMPs seeking healthcare in the public sector. This would be a promising step to improve regulation of malaria treatment in Cambodia, if access to public health services could be improved in remote areas.

A concerning finding of this study was the very low numbers of suspected malaria cases being diagnosed with parasitological blood testing and just under half of MMPs were not aware that malaria should be confirmed by parasitological blood test. In the context of malaria elimination and drug resistance these findings indicate a lot more needs to be done to avoid missing or mistreating cases due to lack of diagnosis [3,25]. Furthermore, there was a significant difference between the numbers of suspected cases being diagnosed appropriately between the MMP subgroups. Forest goers and security personnel were far more likely to be tested, and to have knowledge that malaria should be confirmed by testing, compared to seasonal and construction/ mine workers, suggesting that there was a difference in access to diagnosis between the MMP subgroups. The population movement framework developed by Guyant et al. to better characterise and study MMPs suggested that "access" to healthcare included the following 
components: availability, accessibility, accommodation, affordability and acceptability [17]. In this study, acceptability is unlikely to explain the difference given that trust for the safety and reliability of RDTs was very good for all MMP subgroups. The accessibility of testing may be in part due to which types of healthcare provider were utilised by the different MMP subgroups. For example, forest goers received more tests from VMWs/MMWs/VHVs than any other MMP subgroup and security personnel received more tests from health posts. If seasonal and construction/mine workers utilised healthcare providers who were less likely to provide diagnostic tests, this could explain why these MMPs reported fewer suspected cases being diagnosed. Studies have shown that the private sector in Cambodia is less likely to have RDTs available than the public sector, supporting the theory that the provider may influence access $[14,26]$. However, in this study health seeking was only reported in relation to receiving parasitological blood testing, so it is not possible to determine overall which providers were more likely to supply RDTs. Affordability may have also been an influencing factor given that studies have suggested that MMPs are more likely to incur out of pocket expenses for health services, in particular some groups of MMPs may suffer from this more than others, making them unlikely to want to pay additional fees for diagnostic tests [24]. Understanding the reasons behind the poor access to diagnosis is critical to be able to remove barriers which particular MMP subgroups face. The importance of quality of care cannot be overstated when the country is trying to achieve elimination.

Among the MMPs who were diagnosed positive for malaria, all of them subsequently received treatment, indicating that access to treatment was not an issue. However, the type of treatment was not specified and therefore it is not known whether appropriate antimalarial treatment was provided. In a cross-sectional survey conducted in remote locations in Cambodia, access to both diagnosis and effective treatment was very low. The majority of all artemisinin use was monotherapy, which is of great concern in the context of drug resistance [27]. However, in 2013, the most common anti-malarial to be sold or distributed, in the public and private sector, was Cambodia's first-line ACT. Oral artemisinin monotherapy, which had accounted for $6 \%$ of total anti-malarial market share in 2009 , was no longer reportedly sold or distributed in 2013 [28]. Therefore, more needs to be done to understand what antimalarial treatment MMPs have access to in remote border regions in order to stop this group becoming a driver for drug resistance.

A positive finding in the study was that health seeking behaviour in MMPs was excellent, likely due to the majority of MMPs having a good knowledge of the common symptoms of malaria and an understanding of the consequences of not adhering to the treatment course. Multiple studies in the GMS region have found the knowledge of malaria is associated with improved health seeking, suggesting this is an effective approach to target health seeking behaviour $[16,20]$. Health seeking varies across different MMP subgroups depending on their characteristics, for example, seasonal workers may have reduced health seeking practices due to their increased mobility, whereas migrants who remain for longer in one place are more likely to access health services given that they are more aware of what is available $[20,23]$. Which was a similar pattern found in this study, particularly with forest goers receiving testing from VMWs more than any other MMP subgroup (a phenomenon also noted in a study in Northeast Cambodia), although the differences were not significant [29]. Health seeking between mobile and migrant individuals and their families was found to be significantly associated. Men are usually the decision makers in a typical Cambodian family; therefore their behaviour in relation to seeking healthcare is likely to be highly influential on the rest of the family $[30,31]$. This suggests that interventions targeting MMP health seeking behaviour may also positively impact on their families. This is particularly important given that MMPs have been reported to import malaria back to the place of residence, and areas with higher numbers of migrants have higher incidence of malaria.

Dissemination of this information is important to inform future research and interventions targeting MMPs and other high-risk hardto-reach groups. Subsequent diffusion of successful interventions and innovations must be carried out to provide meaningful impact of findings.

\section{Limitations}

Data collection in this study was conducted through questionnaires and is therefore subject to recall and desirability biases. The recall period was set to three months to try to limit this issue. The information to do with health seeking and related knowledge were individual sections in a wider questionnaire, therefore the depth of information that could be collected if solely looking at these two topics was not possible. This resulted in information about health seeking to providers in general (not just providers of diagnostic testing) and some other small topics were not collected. Finally, due to the nature of the MMP, truly random sampling was not possible. However, great efforts were made to choose samples which were representative of their MMP subgroup and were distributed evenly throughout the selected locations.

\section{Conclusion}

MMPs are at an elevated risk of malaria compared to the general population, and this risk even varies within MMPs depending on the characteristics of the subgroup. Although health seeking behaviour was reportedly good for all MMP subgroups particularly to health centres, district hospitals and volunteers, barriers in access to care were significantly different. This highlights that the MMP can no longer be treated as a homogenous group when targeted by malaria interventions. The barriers that different MMP subgroups face in accessing diagnosis need to be addressed in order to effectively treat these high-risk populations. Further research must therefore be conducted to understand why these differences have occurred.

\section{Acknowledgement}

This work was supported by the Global Fund to Fight Tuberculosis, AIDS and Malaria (GFTAM) through the United Nations Office for Project Services (UNOPS) with technical support from Malaria Consortium.

\section{References}

1. WHO (2013) Planning meeting for operational research on malaria elimination Meeting Report: Global Malaria Programme.

2. Maude RJ, Nguon C, Ly P, Bunkea T, Ngor P, et al. (2014) Spatial and tempora epidemiology of clinical malaria in Cambodia 2004-2013. Malar J 13: 385. [Crossref]

3. Ministry of Health (2011) The National Strategic Plan for Elimination of Malaria in the Kingdom of Cambodia. 2011-2025.

4. Canavati E, Lawford L S, Fatunmbi B S, Lek D, Top-Samphor N, et al. (2016) Establishing research priorities for malaria elimination in the context of the emergency response to artemisinin resistance framework-the Cambodian approach. Malaria Journal 15: 120.

5. Wai KT, Kyaw MP, Oo T, Zaw P, Nyunt MH, et al. (2014) Spatial distribution, work patterns, and perception towards malaria interventions among temporary mobile/ migrant workers in artemisinin resistance containment zone. BMC Public Health 14: 463. [Crossref] 
6. Noedl H, Se Y, Schaecher K, Smith BL, Socheat D, et al. (2008) Evidence of artemisininresistant malaria in western Cambodia. $N$ Engl J Med 359: 2619-2620. [Crossref]

7. Karunajeewa HA (2015) Parasite clearance after malaria therapy: staying a step ahead of drug resistance. BMC Medicine 13: 251

8. Verschuere J, Decroo T, Lim D, Kindermans JM, Nguon C, et al. (2017) Local constraints to access appropriate malaria treatment in the context of parasite resistance in Cambodia: a qualitative study. Malaria Journal 16: 81

9. Cotter C, Sturrock HJ, Hsiang MS, Liu J, Phillips AA, et al. (2013) The changing epidemiology of malaria elimination: new strategies for new challenges. Lancet 382: 900-911. [Crossref]

10. Chen I, Thanh HNT, Lover A, Thao PT, Luu TV, et al. (2017) Malaria risk factors and care-seeking behaviour within the private sector among high-risk populations in Vietnam: a qualitative study. Malar J 16: p. 414. [Crossref]

11. Leang T, Bunkea L, Dysoley C, Nguon (2010) Cambodia Containment Surveys 2009 and 2010.

12. Zhou G, Sirichaisinthop J, Sattabongkot J, Jones J, Bjørnstad ON, et al. (2005) Spatiotemporal distribution of Plasmodium falciparum and p. Vivax malaria in Thailand. Am J Trop Med Hyg 72: 256-262. [Crossref]

13. Edwards HM, Canavati SE, Rang C, Ly P, Sovannaroth S (2015) Novel Cross-Border Approaches to Optimise Identification of Asymptomatic and Artemisinin-Resistant Plasmodium Infection in Mobile Populations Crossing Cambodian Borders. PloS One 10: e0124300. [Crossref]

14. Ly P, Thwing J, McGinn C, Quintero CE, Top-Samphor N, et al. (2017) The use of respondent-driven sampling to assess malaria knowledge, treatment-seeking behaviours and preventive practices among mobile and migrant populations in a setting of artemisinin resistance in Western Cambodia. Malar J 16: 378 [Crossref]

15. Durnez L, Mao S, Denis L, Roelants P, Sochantha T, et al. (2013) Outdoor malaria transmission in forested villages of Cambodia. Malar J 12: 329. [Crossref]

16. Win AYN, Maung TM, Wai KT, Oo T, Thi A, et al. (2017) Understanding malaria treatment-seeking preferences within the public sector amongst mobile/migrant workers in a malaria elimination scenario: a mixed-methods study. Malar $J$ 16: 462. [Crossref]

17. Guyant P, Canavati SE, Chea N, Ly P, Whittaker MA, et al. (2015) Malaria and the mobile and migrant population in Cambodia: a population movement framework to inform strategies for malaria control and elimination. Malar J 14: 252. [Crossref]

18. Khamsiriwatchara P, Wangroongsarb J, Thwing J, Eliades, Satimai W, et al. (2011) Respondent-driven sampling on the Thailand-Cambodia border. I. Can malaria cases be contained in mobile migrant workers. Malar J 10: 120. [Crossref]
19. Canavati N, Chea P, Guyant A, Roca-Feltrer (2013) Strategy to address migrant and mobile populations for malaria elimination in Cambodia. MMP Strategy 2013

20. Canavati SE, Quintero CE, Lawford HLS, Yok S, Lek D, et al. (2016) High mobility, low access thwarts interventions among seasonal workers in the Greater Mekong Subregion: lessons from the malaria containment project. Malaria Journal 15: 434.

21. Dysoley L, Kaneko A, Eto H, Mita T, Socheat D, et al. (2008) Changing patterns of forest malaria among the mobile adult male population in Chumkiri District, Cambodia. Acta Trop 106: 207-212. [Crossref]

22. CNM (2010) Cambodia Malaria Survey.

23. Wangroongsarb P, Satimai W, Khamsiriwatchara A, Thwing J, Eliades JM, et al (2011) Respondent-driven sampling on the Thailand-Cambodia border. II. Knowledge, perception, practice and treatment-seeking behaviour of migrants in malaria endemic zones. Malar J 10: 117. [Crossref]

24. McMichael C, Healy J (2017) Health equity and migrants in the Greater Mekong Subregion Glob Health Action 10: 1271594. [Crossref]

25. Duru V, Witkowski B, Ménard D (2016) Plasmodium falciparum Resistance to Artemisinin Derivatives and Piperaquine: A Major Challenge for Malaria Elimination in Cambodia. Am J Trop Med Hyg 95: 1228-1238. [Crossref]

26. Poyer S, Shewchuk T, Tougher S, Ye Y, Mann AG, et al. (2015) Availability and price of malaria rapid diagnostic tests in the public and private health sectors in 2011: results from 10 nationally representative cross-sectional retail surveys. Trop Med Int Health 20: 744-756. [Crossref]

27. Yeung S, Van Damme W, Socheat D, White NJ, Mills A, et al. (2008) Access to artemisinin combination therapy for malaria in remote areas of Cambodia. Malar $\mathrm{J} 7$ : 96. [Crossref]

28. ACT watch Group, Novotny J, Singh A, Dysoley L, Sovannaroth S, et al. (2016) Evidence of successful malaria case management policy implementation in Cambodia: results from national ACT watch outlet surveys. Malar J 15: 194. [Crossref]

29. Peeters Grietens K, Gryseels C, Dierickx S, Bannister-Tyrrell M, Trienekens S, et al. (2015) Characterizing Types of Human Mobility to Inform Differential and Targeted Malaria Elimination Strategies in Northeast Cambodia. Sci Rep 5: 16837. [Crossref]

30. Samandari G, Speizer IS, O'Connell K (2010) The role of social support and parity in contraceptive use in Cambodia. Int Perspect Sex Reprod Health 36: 122-131. [Crossref]

31. Bopha H, Kroesna K, Seng S, Bunneth B (2011) The role of gender in decision-making in investing and managing the household livelihood in Stug Chinit Irrigation Common Area, Kampong Thom Province. Int J Environ Rural Dev

Copyright: $(2019$ Kheang ST. This is an open-access article distributed under the terms of the Creative Commons Attribution License, which permits unrestricted use, distribution, and reproduction in any medium, provided the original author and source are credited. 